\title{
GPPS-BJ-2019-0052
}

\section{KEPLER: An integrated simulating system for efficient industrial design and optimization}

\author{
Yi Lu \\ Dimaxer Technology LTD \\ luyi@dimaxer.com \\ Cambridge, UK
}

\author{
Kai Liu \\ Dimaxer Technology KK \\ kai@dimaxer.com \\ Osaka, Japan
}

\section{ABSTRACT}

This paper describes the fundamental numerical schemes, objectives, structure and current capabilities of the KEPLER simulating system. This computational analysis and design software collection is aim to be designed and developed to solve wide range of industrial simulations in analysis and optimization tasks for industrial designers. KEPLER integrates pre-processing(automatic mesh generation), simulation and post-processing by using the "In-memory database" technology, which provide one-step solution for complete simulations without switching between different softwares/tools, also enables real-time reading/editing configurations and extracting results during the simulation by multi-users which could increase the working and collaboration efficiency dramatically.

KEPLER provides unsteady solutions for compressible and incompressible flows(CFD) based on innovative high order Kinetic Energy Preserving flux reconstruction method with space-time expansion as time marching method(STEKEP-FR). FR method is a localized stencil numerical scheme which constructs high order discretization completely in-cell, this method is very efficient for its simple differential formulation. The KEP method preserve the order of accuracy and enhance the nonlinear stability of FR discretization without increasing distinct computing cost. The STE method enables time-accurate local time-stepping, which means each cell use its local optimal time-step instead of the globally smallest time-step during the time-marching process, and the local time-step could be adaptive for stability and any other dynamic process. Because of the locality of STE-FR-FR method on both space and time, time-adaptive non-conformal and overset mesh are using seamless and efficiently on KEPLER, which enable the abilities for simulating complex moving/deforming geometries and wide range of dynamic flow problems.

The computing efficiency, accuracy, stability of convection calculations, and overset performance is investigated in this work which are all critical issues for the ability and Competitiveness of Kepler code.

\section{INTRODUCTION}

Over the last several decades, Computational fluid dynamics(CFD) has progressed rapidly with the increasing computational abilities and continued development on numerical algorithms, and plays a critical role for modern wide range of industrial products design process. However, both popular commercial CFD software and productive inhouse codes still are inadequate to provide fast and high fidelity simulations to meet the requirements in industry. The main required capabilities of next generation CFD has been summarized by NASA report CFD 2030 vision study ${ }^{[1]}$ as:

1. Advanced physical-based predictive modeling;

2. Management and Tolerance for errors and uncertainties;

3. Higher automation in all steps;

4. Making use of most capable HPC hardwares;

5. Flexible to perform both research work and productive industrial design jobs as data-base applications;

6. Seamlessly integrated simulation system for multidisciplinary analysis and optimizations.

In order to meet these requirements, this paper introduces the integrated simulating system with following features:

1. Integrated data-structure for pre-processing, adaptive simulation and post-processing based on uniform "In-memory database".

2. Advanced numerical algorithm, the STE-KEP-FR ${ }^{[2]}$ method, which combine knowledges of high order flux reconstruction discretization on hybrid mixed unstructured meshes, improved non-linear stability by implementing splitform on convective term, very high time-marching efficiency by using time-accurate local time-stepping time marching method, result in the solver KEPLER. KEPLER is able to use hybrid unstructured meshes with non-conformal/overset, to solve wide range of flow problems by adopting innovative shock-capturing method for high speed flow simulations and $\mathrm{EDAC}^{[4]}$ formulations INS for efficient incompressible flow simulations. 
3. Implementation on different many core computing systems based on uniform "Server-coprocessors" framework, which could break memory bottleneck of modern GPUs by using "Real-time loading" method ${ }^{[3]}$;
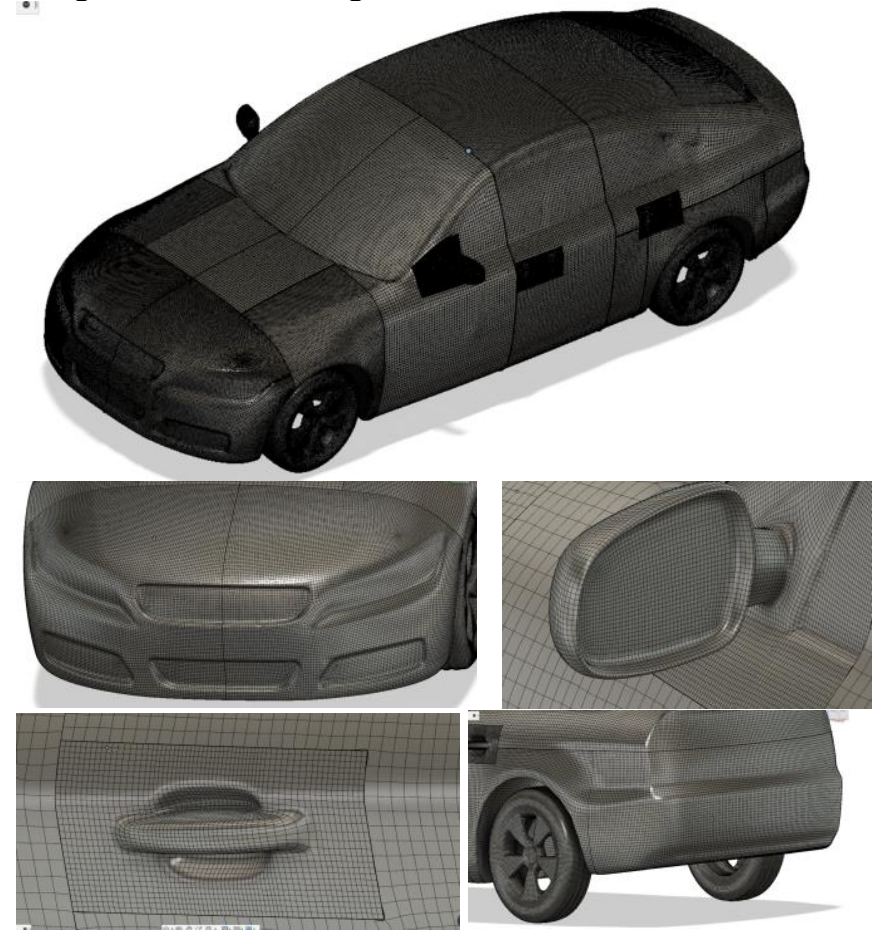

Figure 1: Surface mesh for DriVaer case generate form T-Spline CAD files

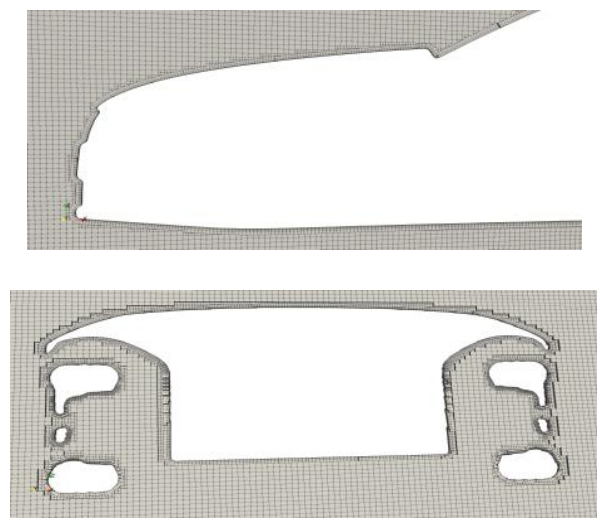

Figure 2: Near-wall overset mesh for DriVaer real geometry case

The pre-processing (mesh generation) start from T-Spline form CAD format and generate separate non-conformal and water-tight surface patch which all consist of pure quadrilateral elements as shown in Figure 1 (a) (e), and extrude boundary layer mesh and overlapped with background tree-based cells illustrated in Figure 2.

In order to achieve the very high efficient high fidelity simulations which is fast enough to be used for industrial design, some studies has been performed to investigate the accuracy, stability, overset accuracy and computing speed of this solver in this paper.
This paper is structured as follows. First, the architecture of KEPLER is introduced with the kernel data structure based on "in-memory data". Next, the fundamental numerical schemes STE-KEP-FR in reviewed, followed by the implementation on GPUs. Afterword some numerical test and analysis are presented. Finally some conclusions are drawn.

\section{ARCHITECTURE OF KEPLER}

The architecture of KEPLER code is illustrated in Fig.3, the fundamental numerical scheme STE-KEP-FR has following main features:

1.Simple, flexible and efficient spatial discretization by using flux reconstruction(FR) method and is able to achieve arbitrary orders of accuracy on hybrid unstructured meshes;

2.Enhanced non-linear stability and main accuracy by using split form kinetic energy preserving(KEP) scheme on convective term, which is critical for high fidelity simulations on high fidelity simulations for practical problems;

3. Allows time-accurate local time-stepping by using innovative "predictor-corrector" type integration method. This method is able to achieve 1 to 2 orders of magnitude speed up ratio for realistic unsteady simulations compared to conventional uniform time-stepping methods.

As shown in the KEPLER-SOLVER code structure in Fig.2, equation definition and equations of states(fluid definition) are used as template parameters, which means this code is open and very easy to add new equations as long as the flux calculation is well defined, all features introduced above and all functionalities in this work is applied.
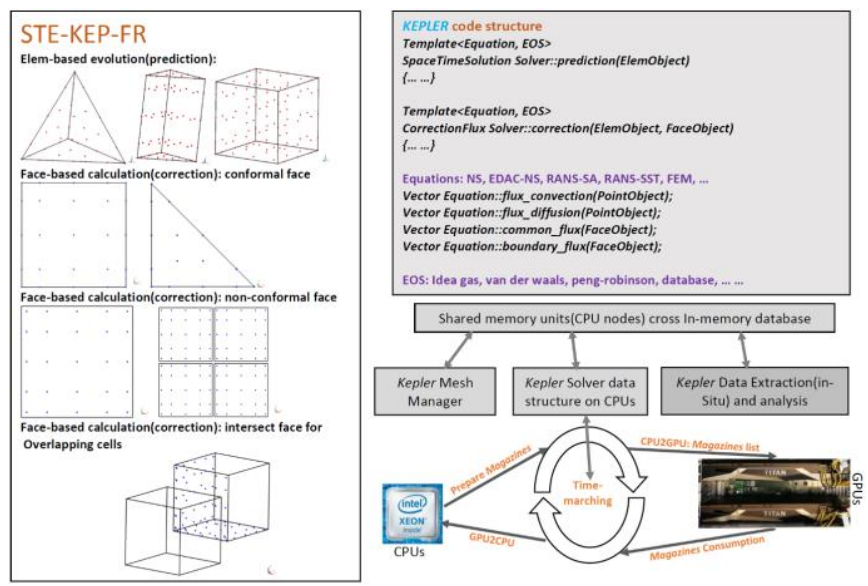

Figure 3: Architecture of KEPLER solver 


\section{Fundamental Numerical Schemes}

The spatial discretization method of STE-KEP-FR is implemented completely in-cell, as shown in Fig.4, Lagrange polynomials are constructed on the solution points with order of accuracy up to $6^{\text {th }}$ order for practical simulations in this work. Element based operations are performed on solution points, where face-based calculate is performed on collocated flux points on each element face coincidence with part of solution points. Details of complete introduction could be found in [2].
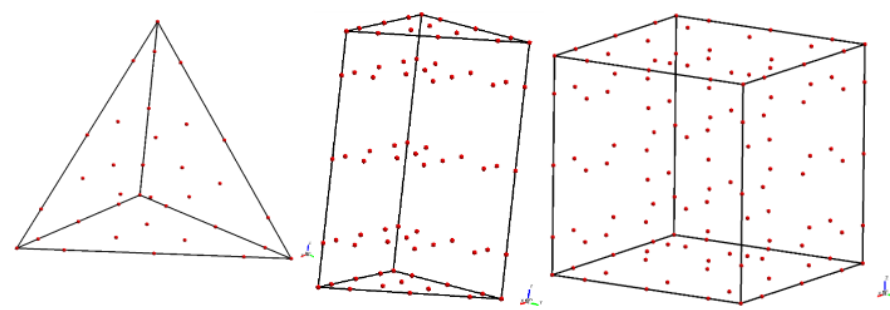

\section{Figure 4: Solution point (SP, red dots) locations for 3D elements, $K=4$ (5th order)}

In order to simply review the STE-KEP-FR method, firstly define the coordinate transformation from cartesian to computational domain

$$
\begin{gathered}
\widehat{U}=|\boldsymbol{J}| U \\
F^{\xi}=|\boldsymbol{J}|\left(\xi_{x} F^{x}+\xi_{y} F^{y}+\xi_{z} F^{z}\right) \\
F^{\eta}=|\boldsymbol{J}|\left(\eta_{x} F^{x}+\eta_{y} F^{y}+\eta_{z} F^{z}\right) \\
F^{\zeta}=|\boldsymbol{J}|\left(\zeta_{x} F^{x}+\zeta_{y} F^{y}+\zeta_{z} F^{z}\right)
\end{gathered}
$$

Where $U$ is conservative variable, $\vec{F}^{x}=\left(F^{x}, F^{z}, F^{z}\right)$ and $\vec{F}^{\xi}=\left(F^{\xi}, F^{\eta}, F^{\zeta}\right)$ are fluxes calculated on cartesian coordinates and computational coordinates, respectively. Jacobian matrix $\boldsymbol{J}$ is given by

$$
\boldsymbol{J}=\left|\begin{array}{lll}
x_{\xi} & x_{\eta} & x_{\zeta} \\
y_{\xi} & y_{\eta} & y_{\zeta} \\
z_{\xi} & z_{\eta} & z_{\zeta}
\end{array}\right|
$$

Time-dependent hyperbolic ODE in computational domain is written as

$$
\frac{\partial \widehat{U}}{\partial t}+\nabla^{\xi} \cdot \overrightarrow{\boldsymbol{F}}^{\xi}=\frac{\partial \widehat{\boldsymbol{U}}}{\partial t}+\frac{\partial \boldsymbol{F}^{\xi}}{\partial \xi}+\frac{\partial \boldsymbol{F}^{\eta}}{\partial \eta}+\frac{\partial \boldsymbol{F}^{\zeta}}{\partial \zeta}=0
$$

Fig 1. illustrates the solution points for $4^{\text {th }}$ order polynomial $\left(5^{\text {th }}\right.$ order $)$ on different types of $3 \mathrm{D}$. The uniform $\mathrm{FR}(\mathrm{CPR})$ discretization for $j$-th solution point of $i$-th element are given as

$$
\begin{gathered}
\frac{\partial \widehat{U}_{i, j}}{\partial t}+\left(\nabla^{\xi} \cdot \overrightarrow{\boldsymbol{F}}^{\xi}\left(\boldsymbol{U}_{i}\right)\right)_{i, j}+\sum_{s=1}^{N_{s}} \sum_{f=1}^{N_{f}} \alpha_{j, s, f}\left(\left.\widetilde{\boldsymbol{F}}^{\xi}\right|_{n}-\right. \\
\left.\left.\overline{\boldsymbol{F}}^{\xi}\right|_{n}\right)_{j, s, f}=0
\end{gathered}
$$

Where $N_{s}$ is number of element interfaces and $N_{f}$ equals the number of flux points on the interface. $\overline{\boldsymbol{F}}$ denote the local flux calculated by polynomials constructed in cell, while $\widetilde{\boldsymbol{F}}$ denote common fluxes which commonly take the form of Riemann fluxes for non-linear inviscid flux and central average for viscous part. $\left.\boldsymbol{F}\right|_{n}=\overrightarrow{\boldsymbol{F}} \cdot \vec{n}$ where $\vec{n}$ is outer normal unit vector on each flux point. The difference between common flux and the outer normal projection of local flux
$\left.\widetilde{\boldsymbol{F}}^{\xi}\right|_{n}-\left.\overline{\boldsymbol{F}}^{\xi}\right|_{n}$ is called "correction flux", which is used to update the DOFs by exchanging information with adjoint elements in a conservative way. Details of the FR coefficients $\alpha$ and flux divergence part $\left(\nabla^{\xi} \cdot \overrightarrow{\boldsymbol{F}}^{\xi}\left(\boldsymbol{U}_{i}\right)\right)$ could be found in [9][10]. The following split form Split $_{F e}$ is used to calculate convective term(without pressure) of divergence part in Navier-Stokes equations

$$
\nabla^{\xi} \cdot \overrightarrow{\boldsymbol{F}}^{\xi}\left(\boldsymbol{U}_{i}\right)=\frac{1}{2} \frac{\partial}{\partial \xi}(\widehat{U} \phi)+\frac{1}{2} \phi \frac{\partial}{\partial \xi}(\widehat{U})+\frac{1}{2} \widehat{U} \frac{\partial}{\partial \xi}(\phi)
$$

Which increase the nonlinear stability by its kinetic energy preserving property.

Rewrite $\mathrm{Eq}(4)$. as

$$
\frac{\partial U_{i, j}}{\partial t}=\mathbb{R}_{i, j}^{D i v}\left(\boldsymbol{U}_{i}\right)+\sum_{s=1}^{N_{s}} \sum_{f=1}^{N_{f}} \mathbb{R}_{i, j, s, f}^{\text {Cor }}\left(\boldsymbol{U}_{i}, U_{i, s, f}^{a d j}\right)
$$

Where $\mathbb{R}^{\text {Div }}$ and $\mathbb{R}^{\text {Cor }}$ are "flux divergence" part and the linear combination of "correction flux" part, respectively. $U_{i, s, f}^{a d j}$ is the conservative variables on adjoint point(same location but in another cell) of flux point $p_{i, s, f} . \mathbb{R}^{\text {Div }}$ and $\mathbb{R}^{\text {Cor }}$ are given as

$$
\begin{aligned}
\mathbb{R}_{i, j}^{D i v} & =-\frac{1}{|\boldsymbol{J}|_{i, j}}\left(\nabla^{\xi} \cdot \vec{F}^{\xi}\left(\boldsymbol{U}_{i}\right)\right)_{i, j} \\
\mathbb{R}_{i, j, s, f}^{C o r} & =-\frac{1}{|\boldsymbol{J}|_{i, j}} \alpha_{j, s, f}\left(\left.\widetilde{\boldsymbol{F}}^{\xi}\right|_{n}-\left.\overline{\boldsymbol{F}}^{\xi}\right|_{n}\right)_{j, s, f}
\end{aligned}
$$

Integrating Eq.(6) over $t \in\left[t^{n}, t^{n+1}\right]$, one obtains

$$
\begin{gathered}
U_{i, j}^{n+1}-U_{i, j}^{n}= \\
\int_{t^{n}}^{t^{n+1}} \mathbb{R}_{i, j}^{\text {Div }}\left(\boldsymbol{U}_{i}\right)+\sum_{s=1}^{N_{s}} \sum_{f=1}^{N_{f}} \mathbb{R}_{i, j, s, f}^{\text {Cor }}\left(\boldsymbol{U}_{i}, U_{i, s, f}^{a d j}\right) d t
\end{gathered}
$$

Construct a local space-time approximation $\boldsymbol{v}_{i}=\boldsymbol{v}\left(\vec{x}_{i}, t\right)$, for $t \in\left[t^{n}, t^{n+1}\right]$ by solving the following time-dependent ODE

$$
\frac{d v_{i, j}}{d t}=\mathbb{R}_{i, j}^{D i v}\left(v\left(\vec{x}_{i}, t\right)\right)
$$

Where the initial value $\boldsymbol{v}\left(\vec{x}_{i}, t=0\right)=\boldsymbol{U}_{i}^{n}(\vec{x})$, the continuous Runge-Kutta method ${ }^{[22]}$ is adopted to solve (9). Integrating Eq.(9) for $t \in\left[t^{n}, t^{n+1}\right]$, one obtains

$$
\int_{t^{n}}^{t^{n+1}} \mathbb{R}_{i, j}^{D i v}\left(\boldsymbol{v}_{i}\right) d t=v_{i, j}\left(t^{n+1}\right)-U_{i, j}^{n}
$$

Taking the space-time polynomial $\boldsymbol{v}\left(\vec{x}_{i}, t\right)$ as a local predictor, and the combination of correction flux $\mathbb{R}^{\text {Cor }}$ as corrector, substituting Eq.(10) into Eq.(8) which results in the space time expansion of flux reconstruction(STE-KEPFR) scheme as

$$
\begin{gathered}
U_{i, j}^{n+1}=v_{i, j}\left(t^{n+1}\right)+ \\
\int_{t^{n}}^{t^{n+1}} \sum_{s=1}^{N_{s}} \sum_{f=1}^{N_{f}} \mathbb{R}_{i, j, s, f}^{C o r}\left(\boldsymbol{v}_{i}\left(t^{n+1}\right), v_{i, s, f}^{a d j}\left(t^{n+1}\right)\right) d t
\end{gathered}
$$

In order to inclusive wider range of element connections on STE time-marching framework, rewrite the above equation as

$$
\begin{gathered}
U_{i, j}^{n+1}=v_{i, j}\left(t^{n+1}\right)+ \\
\sum_{s=1}^{N_{s}} \sum_{f=1}^{N_{f}} \int_{t^{n}}^{t^{n+1}} \mathbb{R}_{i, j, s, f}^{\operatorname{Cor}}\left(\boldsymbol{v}_{i}\left(t^{n+1}\right), v_{i, s, f}^{a d j}\left(t^{n+1}\right)\right) d t
\end{gathered}
$$

Which means the time-integrations of correction flux could be performed on each flux point rather than a whole element interface, this is a very important property to support flexible mesh motions such as non-conformal and overset grids which will be introduced later. Numerical time integration is performed as follow:

$$
\begin{gathered}
U_{i, j}^{n+1}=v_{i, j}\left(t^{n+1}\right)+\sum_{s=1}^{N_{s}} \sum_{f=1}^{N_{f}} \sum_{q p}^{N_{q p}^{t}} \Delta t . \\
w_{q p}^{t} \mathbb{R}_{i, j, s, f}^{C o r}\left(\boldsymbol{v}_{i}\left(t_{q p}\right), v_{i, s, f}^{a d j}\left(t_{q p}\right)\right)
\end{gathered}
$$


Where $N_{q p}^{t}$ is the number of time quadrature points(1D nondimensional Gauss-Quadrature), $w_{q p}^{t}$ is the non-dimensional quadrature weight on time quadrature point $q p, t_{q p}=t^{n}+$ $\Delta t \cdot \tau_{q p}$ is the physical time corresponding to nondimensional 1D quadrature point value $\tau_{q p} \in[0,1]$.

The actual time-marching process of STE-KEP-FR method is:

1. For $i$-th element at physical time point $T_{i}$, calculate local time step $\Delta t_{i}$

2. Perform "prediction" to construct local space time polynomials $\boldsymbol{v}\left(\vec{x}_{i}, \tau\right)$, while $t(\tau)=T_{i}+\Delta t_{i} \cdot \tau, \tau \in[0,1]$ 3. On each flux point $F P_{i, s, f}$, investigate the adjoint point $F P_{i, s, f}^{a d j}$, if the time interval $\left[T_{i, s, f}^{a d j}, T_{i, s, f}^{a d j}+\Delta t_{i, s, f}^{a d j}\right]$ of the space-time polynomial on the element contain $F P_{i, s, f}^{a d j}$, fulfill the condition that $t\left(\tau_{q p}\right) \in\left[T_{i, s, f}^{a d j}, T_{i, s, f}^{a d j}+\Delta t_{i, s, f}^{a d j}\right]$, calculate the time integration of correction flux $\Delta t \cdot w_{q p}^{t}\left(\left.\widetilde{\boldsymbol{F}}^{\xi}\right|_{n}-\right.$ $\left.\left.\overline{\boldsymbol{F}}^{\xi}\right|_{n}\right)_{j, s, f}$

4. Investigate all flux points on each side $s$, if $\sum_{s=1}^{N_{s}} \sum_{f=1}^{N_{f}} \sum_{q p}^{N_{q p}^{t}} \Delta t \cdot w_{q p}^{t}\left(\left.\widetilde{\boldsymbol{F}}^{\xi}\right|_{n}-\left.\overline{\boldsymbol{F}}^{\xi}\right|_{n}\right)$ has been finished, take the result of Eq.(9) institute $\operatorname{Eq(13)}$ to update to $U_{i, j}^{n+1}\left(T_{i}+\Delta t_{i}\right)$, then go to step one and start again from physical time point $T_{i}+\Delta t_{i}$.

\section{Implementation on GPUs}

All numerical validations are performed on two workstations as shown in Fig.4. Their configurations are listed on Tab.1, it could be found that the computing ability of modern GPU is very powerful(up to 6.9TFlops for double precision calculation per GPU), however, the on-site memory is only $12 \mathrm{~GB} / \mathrm{GPU}$ which could not able to undertake enough works with conventional synchronous and uniform jobpartition/communication model.

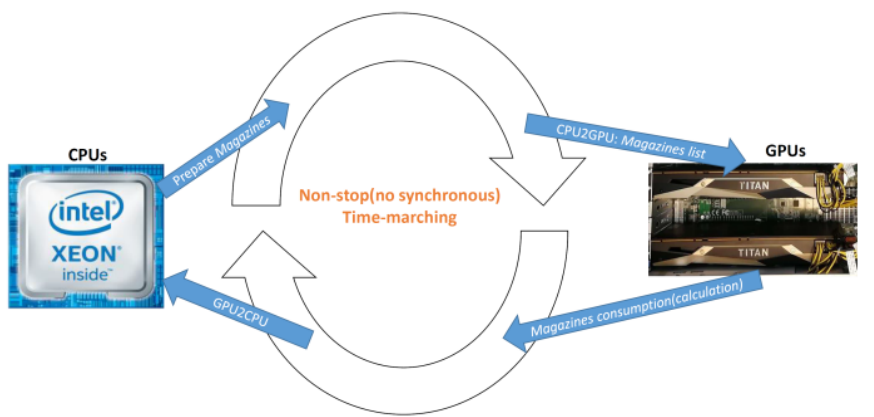

\section{Figure 5: Flowchart of Magazine type computing} model

As reviewed in section III, the time-accurate local time stepping is allowed for STE-KEP-FR method, and a nonstop(no synchronous) time-marching process could be lunched until the terminate of simulations, and a delicate controller was designed to keep casting executive elements(prediction) and element sides(time integration of correction flux and correction) which maintains very good space-time load balancing and nearly real-time efficiency compare to actual computation cost. Based on these works, a new computing model is introduced in this work, which is aimed to use the very limited on-chip GPU memory in a circulated way, and simplify the data-communications between CPUs and GPUs, between different computing nodes. The self-dependent computing package which include all data for calculations is called Magazine in this paper. As shown in Fig 5., there are only four operations for this model:

1. Prepare Magazines on GPU;

2. Transfer Magazine from CPUs to GPUs;

3. Perform calculations on GPUs including: space-time polynomial prediction on each element as $\operatorname{Eq}(9)$, timeintegration of correction flux on executable element side as the second term on RHS of $\mathrm{Eq}(11)$, and final correction as Eq(13).

4. Transfer result data of the "consumption" (calculated) Magazine from GPUs to CPUs;

The above 4 operation could be implemented in an asynchronous mode to hide the time-cost of data transfer between CPUs and GPUs, which means if the computing cost on GPUs is longer than the preparations on GPUs and the cost of data transfer, the parallel scalability is $100 \%$, and also the computing ability of GPUs could be completely used as long as Magazines list is not empty.

In SET-KEP-FR method, as Eq(13), one step time marching method is used which mean only element solution and time integration of correction flux on side need to be prepared in Magazine as

$$
\begin{gathered}
N_{\text {dofs }}=N_{\text {sps }} * 1024^{3} /\left(\left(N_{\text {sps }}+N_{\text {sides }}^{\text {excu }} * N_{\text {fps }}\right) * 8\right) \\
=134.2 \times 10^{6} * N_{\text {sps }} /\left(N_{\text {sps }}+N_{\text {sides }}^{\text {excu }} * N_{\text {fps }}\right)
\end{gathered}
$$

Where 8 means the number of bits for each double precision number, $N_{s p s}$ is the number of solution points on each element, $N_{f p s}$ is the number of flux points on each element side. $N_{\text {sides }}^{\text {excu }}$ is the average number of executive sides for time integration of correction flux or final correction.

For typical $2^{\text {nd }}$ order explicit finite volume method with 4stages Runge-Kutta, assume 1 Magazine contains all minimum data to finish one time-marching steps, the number of average DOFs per GB on single Magazine could be estimated as

$$
\begin{aligned}
N_{\text {dofs }} & =1024^{3} /\left(\left(N_{\text {stages }}+1\right) * 8\right) \\
& =134.2 \times 10^{6} /\left(N_{\text {stages }}+1\right)
\end{aligned}
$$

Tab 1. list the estimated average number of DOFs and Flops per GB for single Magazine, assume all hexahedron elements and $N_{\text {sides }}^{\text {excu }}=3$ (for conformal element interface, the time-integration of correction flux only need to be calculated once for both element sides), and double precision applied for all calculations.

From Tab 1., it can be found that the number of DOFs of FR method is much higher than FVM methods (about one order of magnitude higher in practical), which means much higher computational density in space, and it goes up with higher order of accuracy. In another way, the number of FLOPs(computing operations) increases for higher timeaccuracy(number of CERK stage).

The bandwidth of the data-transfer speed between CPUs and GPUs through PCI-E express is about $11.5 \mathrm{~GB} / \mathrm{s}$ with data size bigger than $64 \mathrm{MB}$. In order to analyze the ratio of computing speed and data transferring speed, study the actual 
speed of high performance GPU card, the highest theoretical computing speed is about 6.9TFlops/s for double precision calculations, however, the practical speed is slower with global memory access and varies for different numerical schemes and code implements. Review Tab 1., 1GB data corresponds to about 0.6 1.5TFlops computing operations for typical high order simulation, the data-transfer for Magazines is higher, or at least comparable to the computing(consumption) speed on GPUs. Though, a delicate design of computing model illustrated in Fig 5., is able to hide the cost of Magazine preparations on CPUs, and the datatransmission between CPUs and GPUs, in asynchronous mode. Details of this computing model can be found in [3].

Table 1: Estimated Average $\boldsymbol{N}_{\text {dofs }}$ and $\boldsymbol{N}_{\text {flops }}$ per GB on single Magazine, assume all hexahedron elements

\begin{tabular}{|c|c|c|c|c|c|}
\hline & $\begin{array}{c}K= \\
1\left(2^{\text {nd }}\right)\end{array}$ & $\begin{array}{c}\boldsymbol{K}= \\
2\left(3^{3 \mathrm{~d}}\right)\end{array}$ & $\begin{array}{c}K= \\
3\left(4^{\text {th }}\right)\end{array}$ & $\begin{array}{c}K= \\
4\left(5^{\text {th }}\right)\end{array}$ & $\begin{array}{c}K= \\
5\left(6^{\text {th }}\right)\end{array}$ \\
\hline $\begin{array}{c}N_{\text {dofs }}( \\
\text { Million } \\
\text { s) }\end{array}$ & $\begin{array}{c}53.7 \\
\mathrm{M}\end{array}$ & $67.1 \mathrm{M}$ & $76.7 \mathrm{M}$ & $83.9 \mathrm{M}$ & $\begin{array}{c}89.5 \\
\mathrm{M}\end{array}$ \\
\hline $\begin{array}{c}N_{\text {flops }} \\
\text { CERK2 } \\
\text { (Giga) }\end{array}$ & 152.1 & 220.2 & 217.4 & 300.2 & 387.3 \\
\hline $\begin{array}{c}N_{\text {flops }}- \\
\text { CERK4 } \\
\text { (Giga) }\end{array}$ & 298 & 432.7 & 428.5 & 593.1 & 766.5 \\
\hline $\begin{array}{c}N_{\text {flops }} \\
\text { CERK6 } \\
\text { (Giga) }\end{array}$ & & & 639.6 & 886 & $\begin{array}{c}1145 . \\
7\end{array}$ \\
\hline $\begin{array}{c}N_{\text {flops }} \\
\text { CERK8 } \\
\text { (Giga) }\end{array}$ & & & & 1178.9 & $\begin{array}{c}1524 . \\
9\end{array}$ \\
\hline
\end{tabular}

\section{Numerical validations and analysis}

Manuscripts must be written in clear English. Both US and UK spellings are acceptable, but should be consistent throughout the manuscript.

The numerical test case is the simulations of standard Tylor-Green vortex decay, with Reynolds number $R e=$ 1600. The initial conditions are specified as

$$
\begin{gathered}
u_{x}^{0}=u_{0} \sin \left(\frac{x}{L}\right) \cos \left(\frac{x}{L}\right) \cos \left(\frac{z}{L}\right) \\
u_{y}^{0}=-u_{0} \cos \left(\frac{x}{L}\right) \sin \left(\frac{x}{L}\right) \cos \left(\frac{z}{L}\right) \\
u_{z}^{0}=0 \\
p^{0}=p_{0}+\frac{\rho_{0} u_{0}^{2}}{16}\left(\cos \left(\frac{2 x}{L}\right)+\cos \left(\frac{2 y}{L}\right)\right)\left(\cos \left(\frac{2 z}{L}\right)+2\right)
\end{gathered}
$$

where $u_{0}=1, p_{0}=100, \rho_{0}=1$. The domain is a periodic with extends $(x, y, z) \in[-\pi L, \pi L]$ and the Reynolds number is 1600 based on $u_{0}$ and $L$ with constant viscosity $\mu=$ $1 / 1600$. The convection time $T_{c}$ is defined as $L / u_{0}$. Most test cases follow the breakdown of the initial conditions up to $30 T_{c}$. Computational mesh is pure uniform hexahedrons with same number of cells along each direction. All simulations were performed on a 4-GPUs(Nvidia Titan V) workstation with dual Intel Xeon Golden 6140 processors.
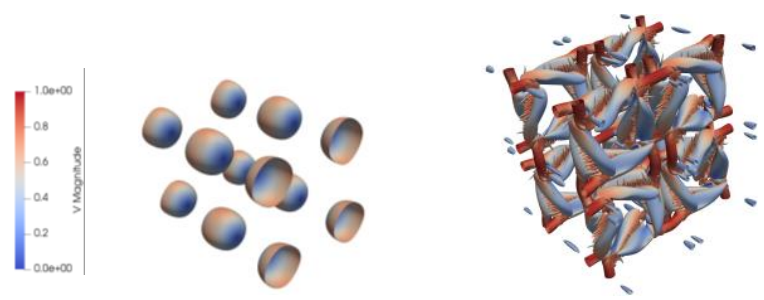

(a) $T \approx 0 s, Q=0.5$

(b) $T=5 T_{c}, Q=1$
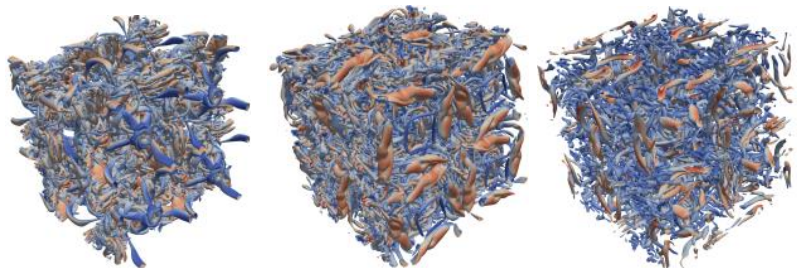

(c) $T=10 T_{c}, Q=1(\mathrm{~d}) T=15 T_{c}, Q=1$ (e) $T=20 T_{c}, Q=1$

Figure 6: Q-criterion iso-surface colored by velocity magnitude

Table 2: Configurations of TGV simulations, $T=$ $30 T_{c}$

\begin{tabular}{|l|c|c|c|c|c|c|c|}
\hline Order & $N_{\text {elems }}$ & $N_{\text {DOFs }}$ & $\begin{array}{l}\text { Equation } \\
\text { state }\end{array}$ & $\begin{array}{l}\text { Mesh } \\
\text { type }\end{array}$ & $N_{C E R K}$ & $N_{q p s}^{t}$ & $\begin{array}{c}\text { Wall } \\
\text { time(h) } \\
\text { GPU } \\
\text { hour }\end{array}$ \\
\hline $\begin{array}{l}2^{\text {nd }} \\
(\mathrm{K}=1)\end{array}$ & $32^{3}$ & $262 \mathrm{~K}$ & NS_KEP & Conf & 2 & 1 & 0.2 \\
\hline $\begin{array}{l}3^{\text {rd }} \\
(\mathrm{K}=2)\end{array}$ & $32^{3}$ & $885 \mathrm{~K}$ & NS_KEP & Conf & 4 & 1 & 0.76 \\
\hline $\begin{array}{l}4^{\text {th }} \\
(\mathrm{K}=3)\end{array}$ & $32^{3}$ & $2.1 \mathrm{M}$ & NS_KEP & Conf & 6 & 2 & 1.26 \\
\hline $\begin{array}{l}5^{\text {th }} \\
(\mathrm{K}=4)\end{array}$ & $32^{3}$ & $4.1 \mathrm{M}$ & NS_KEP & Conf & 8 & 2 & 4.84 \\
\hline $\begin{array}{l}6^{\text {th }} \\
(\mathrm{K}=5)\end{array}$ & $32^{3}$ & $7.08 \mathrm{M}$ & NS_KEP & Conf & 8 & 2 & 8.4 \\
\hline $\begin{array}{l}2^{\text {nd }} \\
(\mathrm{K}=1)\end{array}$ & $96^{3}$ & $7.08 \mathrm{M}$ & NS_KEP & Conf & 2 & 1 & 23.98 \\
\hline $\begin{array}{l}3^{\text {rd }} \\
(\mathrm{K}=2)\end{array}$ & $64^{3}$ & $7.08 \mathrm{M}$ & NS_KEP & Conf & 4 & 1 & 18.3 \\
\hline $\begin{array}{l}6^{\text {th }} \\
(\mathrm{K}=5)\end{array}$ & $16^{3}$ & $885 \mathrm{~K}$ & NS_KEP & Conf & 8 & 2 & 0.53 \\
\hline $\begin{array}{l}6^{\text {th }} \\
(\mathrm{K}=5)\end{array}$ & $32^{3}$ & $7.08 \mathrm{M}$ & NS_KEP & Overs \\
\hline $\begin{array}{l}6^{\text {th }} \\
(\mathrm{K}=5)\end{array}$ & $32^{3}$ & $7.08 \mathrm{M}$ & NS_CR & Conf & 8 & 2 & 8.06 \\
\hline $\begin{array}{l}6^{\text {th }} \\
(\mathrm{K}=5)\end{array}$ & $32^{3}$ & $7.08 \mathrm{M}$ & NS_CR & Overs \\
\hline $\begin{array}{l}3^{\text {rd }} \\
(\mathrm{K}=2)\end{array}$ & $64^{3}$ & $7.08 \mathrm{M}$ & NS_KEP & $\begin{array}{c}\text { Overs } \\
\text { et }\end{array}$ & 4 & 1 & 20.26 \\
\hline
\end{tabular}

Table 3: Comparison of computation efficiency with another high order solver[4] on same resolution

\begin{tabular}{|l|l|l|l|l|l|l|}
\hline Solver & Order & $N_{\text {elems }}$ & $N_{\text {DOFs }}$ & $\begin{array}{l}\text { Physi } \\
\text { cal } \\
\text { time }\end{array}$ & $\begin{array}{l}\text { Computing } \\
\text { platform }\end{array}$ & $\begin{array}{l}\text { Computin } \\
\text { g time (h) }\end{array}$ \\
\hline Cenaero & $\begin{array}{l}6^{\text {th }} \\
(\mathrm{p}=5)\end{array}$ & $38^{3}$ & $\begin{array}{l}11.85 \\
\mathrm{M}\end{array}$ & $10 T_{c}$ & $\begin{array}{l}\text { Intel Xeon } \\
\text { X5675 }\end{array}$ & $\begin{array}{l}10300 \\
\text { CPUh }\end{array}$ \\
\hline
\end{tabular}




\begin{tabular}{|c|c|c|c|c|c|c|}
\hline $\begin{array}{l}\text { Kepler(Di } \\
\text { maxer) }\end{array}$ & $\begin{array}{l}6^{\text {th }} \\
(\mathrm{p}=5)\end{array}$ & $38^{3}$ & $\begin{array}{l}11.85 \\
M\end{array}$ & $10 T_{c}$ & $\begin{array}{l}\text { Nvidia Titan } \\
\text { V }\end{array}$ & $\begin{array}{l}20.4 \\
\text { GPUh }\end{array}$ \\
\hline
\end{tabular}

Computing efficiency:

From the wall time statistic listed in Table. 2, the computing cost is getting lower with increasing the order of accuracy using same number of degree of freedoms, for example, the computational cost of $6^{\text {th }}$ order is only about one quarter compare to $2^{\text {nd }}$ order using the same number of DOFs $192^{3}$. This is a significant progress to push high order schemes for realistic industrial simulations because obviously each high order DOF could achieve much more higher accurate result than lower order DOF, which is discussed later and has been quantified given in [3] using much more simulations as database for analysis, part of the analysis are illustrated in Fig.7 9.

Also compare to another high order discontinuous galerkin(DG) code, by simulation exactly the same case with same configurations as shown in Table. 2, the Kepler code only cost 5.1 hours on a small 4-GPUs workstation, versus 10.3 K CPU hours of Cenaero DG code on a big HPC cluster. This proves the very highly efficient implementation on modern computing system.

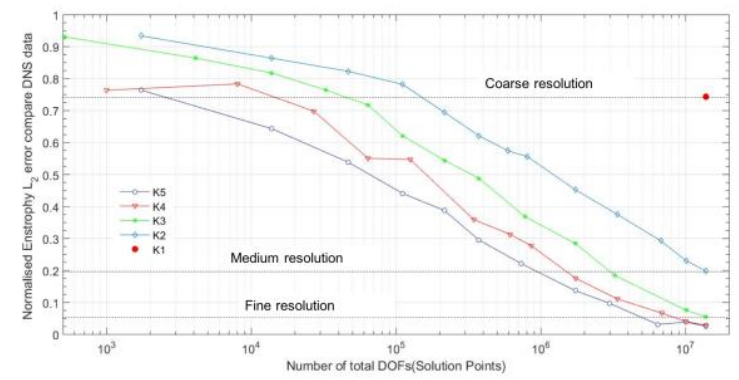

Figure 7: Correlation between Enstrophy prediction error and the number of DOFs for different orders of numerical schemes[2]

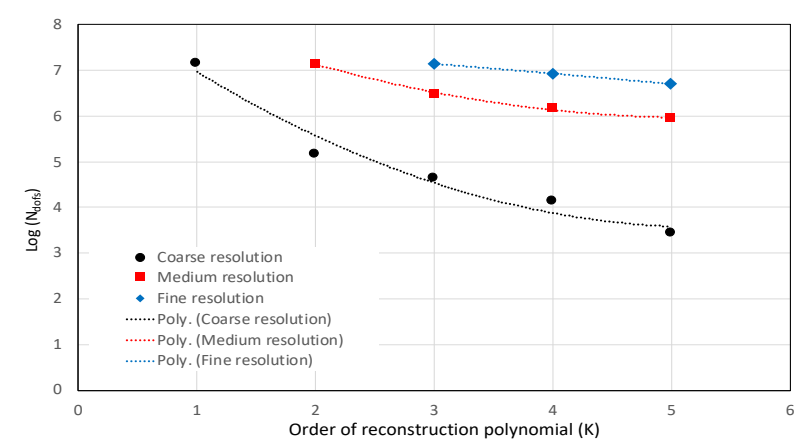

Figure 8: Correlations between the number of DOFs and the order of reconstruction polynomial[2].

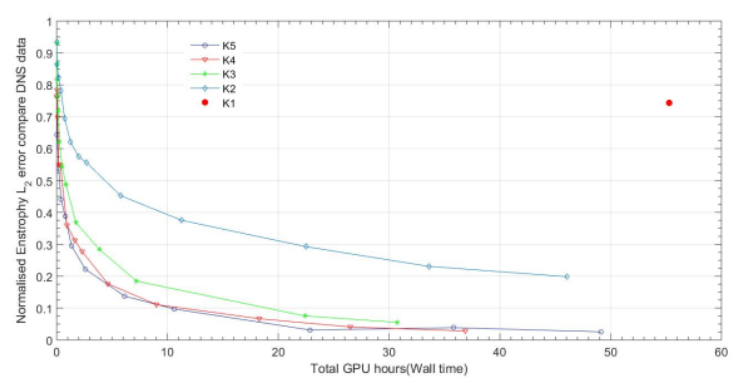

Figure 9: Correlation between Enstrophy prediction error and the computational cost[3]

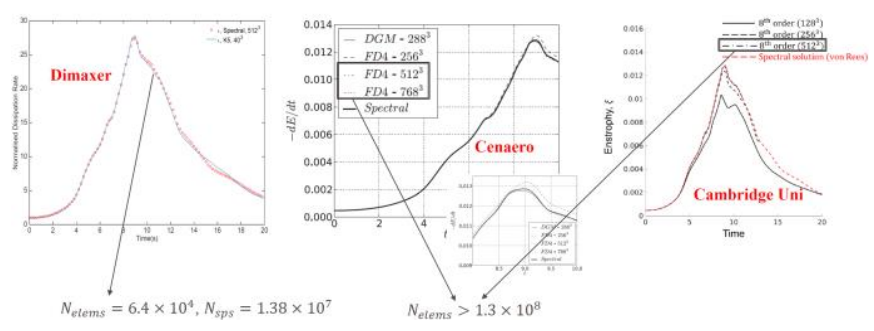

Figure 10: Number of DOFs requirement compare to high order Finite Difference method[5][7]

As proved by Fig.8, the high fidelity simulations using SET-KEP-FR method could use much less(1 2 order of magnitude) DOFs compare to $2^{\text {nd }}$ method, another comparison compare to high order difference methods with comparable resolution has been presented in Fig.10, only 1/10 DOFs and $0.05 \%$ grids are used for $5^{\text {th }}$ order FR method even compare to $8^{\text {th }}$ order FD, no computing cost are available for these high order FD simulations, however, only about 20GPU hours are cost for the Kepler simulation.

\section{Resolution of different orders of accuracy:}

The higher resolution of higher order of accuracy is proved by comparisons of the turbulent Kinetic energy with reliable spectral DNS data, which is drawn in Fig. 7. More importantly, by using the same number of degree of freedoms, higher order simulation still has much better resolution than lower order simulations as shown in Fig. 11 and Fig.9. In particular, the computing cost of higher order simulations per DOF is much cheaper using Kepler code on modern GPUs as listed in Tab. 2, which means the high order simulations is able to provide more accurate result with less computing cost. The much higher ability of the resolution of vortices for higher order simulations is proved by the comparison shown in Fig.12 Fig.13, which indicate the big advantages of Kepler code for high fidelity simulations such as LES and DNS. 

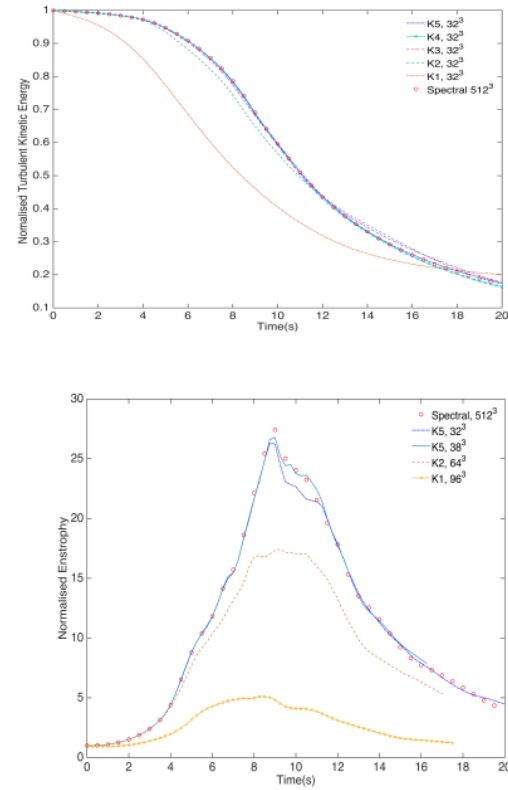

Figure11: Normalized Turbulent Kinetic Energy decay with time for same mesh $32^{3}, K 1 \sim K 5$ (left), and normalized enstrophy for $K 5-32^{\wedge} 3$ and $K 5-$ $38^{3}$ (right)
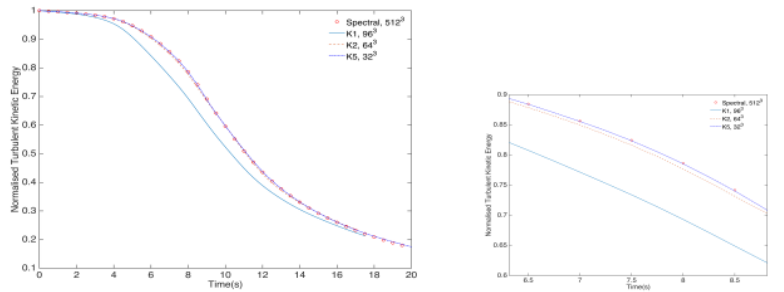

Figure 12: Normalized Turbulent Kinetic Energy decay with time for same DOFs $192^{3}, K 1, K 2$, and K5
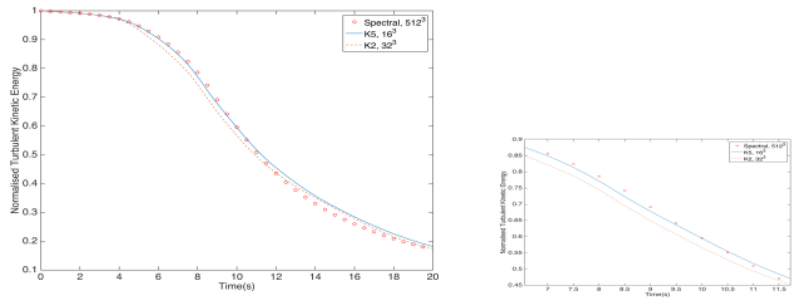

\section{Figure 13: Normalized Turbulent Kinetic Energy} decay with time for same DOFs $96^{3}, K 2$ and $K 5$

Influence of different numerical schemes of divergence part:

Both KEP(kinetic energy preserving) and $\mathrm{CR}$ (Chain-Rule) are used for the calculation of divergence part of the flux reconstruction discretization as $\mathrm{Eq}(7)$. The computational speed of CR is slightly faster than KEP while the result is nearly the same from Fig. 14 for this case.
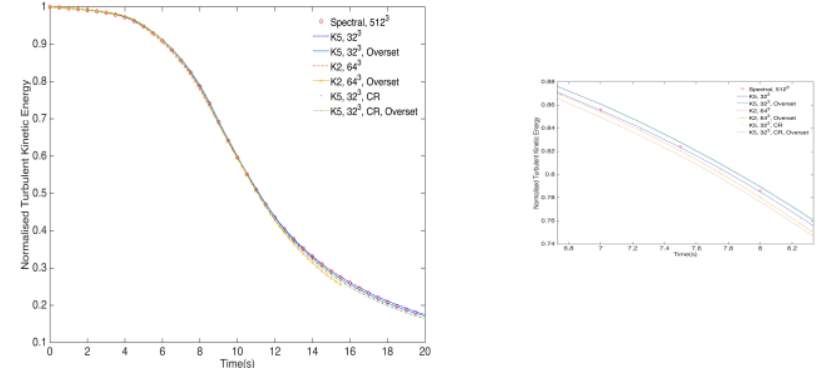

Figure 14: Normalized Turbulent Kinetic Energy decay with time for same DOFs $96^{3}, K 2$ and $K 5$, conformal mesh and overset mesh, Chain-Rule and KEP

\section{Influence of compressibility}

The incompressible NS form named Entropically Damped Artificial Compressibility(EDAC) has been implemented in Kepler $^{[3]}$. Figure 15 lists the detail compassion of both turbulent kinetic energy decay and enstrophy with reference DNS data and a compressible simulation even with slightly higher grid density, all other configurations are exactly the same except Equation of States(EOS). It has been discovered that the slightly error between high order compressible simulations and reference incompressible DNS data after the fully turbulence developed stage $\left(t \geq 9 T_{c}\right)$, is caused by the difference of equations rather than the resolution. The incompressible simulation result match the reference DNS data better than the compressible counterpart even with fewer number of grids, especially at the second half of the overall physical time.
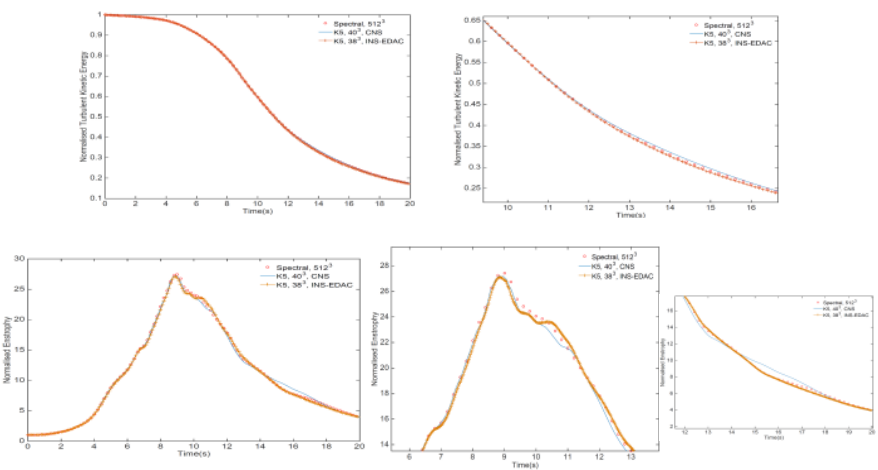

Figure 15: Normalized Turbulent Kinetic Energy $\operatorname{decay}(a \sim b)$, and normalized Enstrophy(c $\sim e)$ for $K 5-40^{3}$ solving compressible NS, and $K 5-38^{3}$ solving EDAC form incompressible NS[3]

Influence of overset and nonconformal

The conformal, overset and nonconformal mesh as shown in Fig. 16, has also been used to investigate the accuracy and computing efficiency. From Fig. 17(a) and Fig. 17 (b), the pressure contour at the same physical time for both conformal and overset mesh has nearly no difference, proves the high order overset simulation maintain the flow flied continuity as using conformal mesh. Fig. 17 (c) and 17(d) present the comparison of turbulent kinetic energy and enstrophy, which demonstrates the adoption of nonconformal 
and overset mesh in high order simulations is able to preserve high resolution.
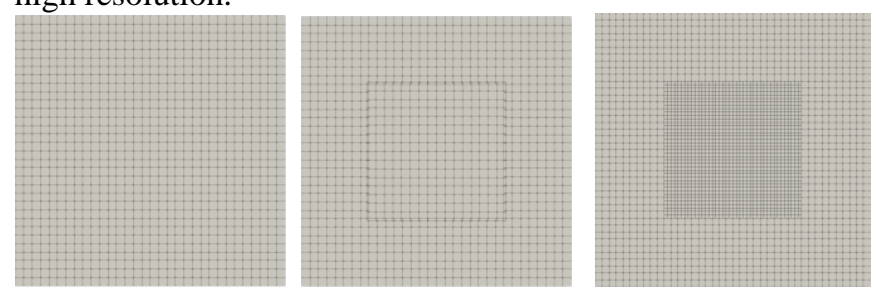

Figure 16: Mesh slice at $Y=\pi$ for $32^{3}$ conformal(left), overset(middle) and $40^{3}$ NonConformal(right)
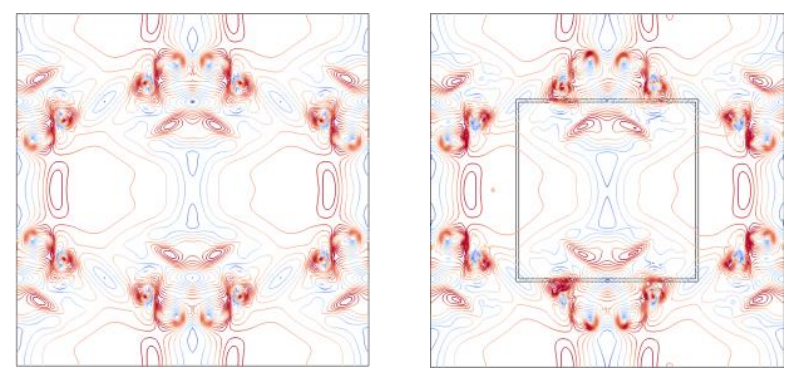

(a) pressure contour at $t=10 T_{c}, K=5,32^{3}$, conformal (b) pressure contour at $t=10 T_{c}, K=5,32^{3}$, overset

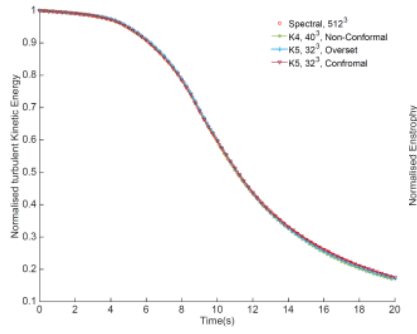

(c) Turbulent kinetic energy

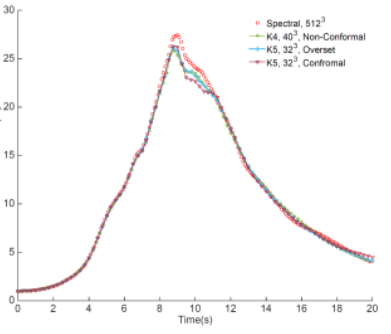

(d) Enstrophy
Figure 17: Result comparison of simulations using conformal, overset and nonconformal meshes

\section{Conclusion}

The detail investigation of computing efficiency, accuracy, overset mesh, stability of two different divergence calculation methods has been performed and discussed in this work. With the combination of innovative computational model, efficient implementation on modern GPUs and advance space and time numerical schemes, Kepler code is potential to perform higher efficient and more accurate simulations compare to widely used 2 nd order solvers. Future works will focus on more practical works on industrial level problems.

\section{Reference papers}

[1] Slotnick J., Khodadoust A., Alonso A., Darmofal D., Gropp W., Lurie E., Maviriplis D. "CFD vision 2030 study: a path to revolutionary computational aerosciences". NASA contractor report, NASA CR-218178, 2014

[2] Lu Y., Cao T., Liu K., "Grid-size requirements for Large Eddy simulations: estimates for different orders of accuracy based on local reconstruction type schemes", AIAA Aviation Paper, 2019
[3] Liu K., Lu Y., "Very fast high order unsteady simulations on GPUs based on a new computational model", AIAA Aviation Paper, 2019

[4] Clausen J., "Entropically damped form of artificial compressibility for explicit simulation of incompressible flow". Phys Rev E 2013; 87:13309-21

[5] Carton C., Hillewaert K., Duponcheel M., Winckelmans G., "Assessment of a discontinuous Galerkin Method for the simulation of vortical flows at high Reynolds number", Int. J. Numer. Meth. Fluid 2014; 74: 469-493

[6] Crabill J., Witherden F. D., Jameson A., “A Parallel Direct Cut Algorithm for High-Order Overset Methods with Application to a Spinning Golf Ball". Sumitted to Journal of Computational Physics.

[7] Przytartski P. J., Wheeler A.P.S., "Accurate prediction of loss using high fidelity methods", ASME paper GT2018-77125 